\title{
Towards the realization on JET of an integrated H-mode scenario for ITER
}

\author{
J. Ongena ${ }^{1, a}$, P. Monier-Garbet ${ }^{2}$, W. Suttrop ${ }^{3}$, Ph. Andrew ${ }^{4}$, M. Bécoulet ${ }^{2}$, \\ R. Budny ${ }^{5}$, Y. Corre ${ }^{6}$, G. Cordey ${ }^{4}$, P. Dumortier ${ }^{1}$, Th. Eich ${ }^{7}$, L. Garzotti ${ }^{8}$, \\ D.L. Hillis ${ }^{9}$, J. Hogan ${ }^{9}$, L.C. Ingesson ${ }^{10}$, S. Jachmich ${ }^{1, \mathrm{c}}$, E. Joffrin ${ }^{2}$, P. Lang ${ }^{7}$, \\ A. Loarte ${ }^{11}$, P. Lomas ${ }^{4}$, G.P. Maddison ${ }^{4}$, D. McDonald ${ }^{4}$, A. Messiaen ${ }^{1}$, \\ M.F.F. Nave ${ }^{12}$, G. Saibene ${ }^{11}$, R. Sartori ${ }^{11}$, O. Sauter ${ }^{13}$, J.D. Strachan ${ }^{14}$, \\ B. Unterberg ${ }^{7}$, M. Valovic ${ }^{4}$, I. Voitsekhovitch ${ }^{15}$, M. von Hellermann ${ }^{10}$, B. Alper ${ }^{4}$, \\ Y. Baranov ${ }^{4}$, M. Beurskens ${ }^{4}$, G. Bonheure ${ }^{1}$, J. Brzozowski ${ }^{14}$, J. Bucalossi ${ }^{2}$, \\ M. Brix ${ }^{7}$, M. Charlet ${ }^{4}$, I. Coffey ${ }^{4}$, M. De Baar ${ }^{4}$, P. De Vries ${ }^{10}$, C. Giroud ${ }^{10}$, \\ C. Gowers ${ }^{4}$, N. Hawkes ${ }^{4}$, G.L. Jackson ${ }^{16}$, C. Jupen ${ }^{14}$, A. Kallenbach ${ }^{3}$, \\ H.R. Koslowski ${ }^{7}$, K.D. Lawson ${ }^{4}$, M. Mantsinen ${ }^{17}$, G. Matthews ${ }^{4}$, F. Milani ${ }^{4}$,

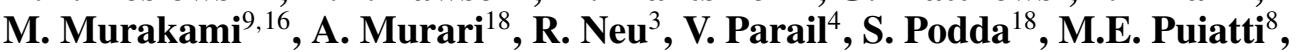 \\ J. Rapp ${ }^{7}$, E. Righi ${ }^{11}$, F. Sartori ${ }^{4}$, Y. Sarazin ${ }^{2}$, A. Staebler ${ }^{3}$, M. Stamp ${ }^{4}$, G. Telesca ${ }^{8}$, \\ M. Valisa ${ }^{8}$, B. Weyssow ${ }^{19}$, K.D. Zastrow ${ }^{4}$ and EFDA-JET Workprogramme \\ contributors $^{\mathrm{c}}$
}

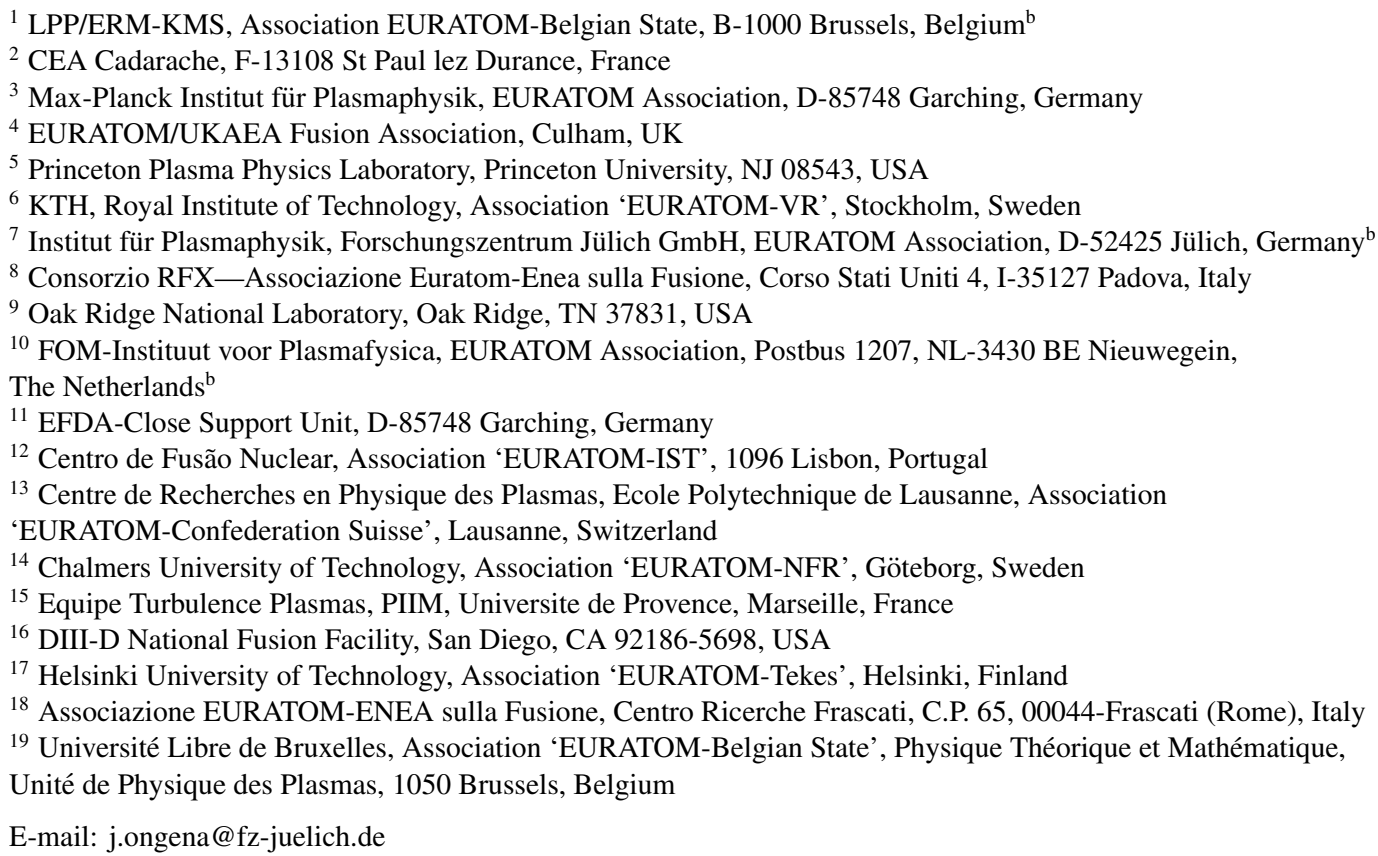

Received 20 November 2002, accepted for publication 4 November 2003

Published 17 December 2003

Online at stacks.iop.org/NF/44/124 (DOI: 10.1088/0029-5515/44/1/015)

\footnotetext{
Abstract

a Researcher at NFSR Belgium.

b Partners in the Trilateral Euregio Cluster (TEC).

c See annex in IAEA 2002 J. Pamela IAEA-CN94/OV-1/1.4.
}

ELMy H-mode experiments at JET in 2000/mid-2002 have focused on discharges with normalized parameters for plasma density, energy confinement and beta similar to those of the ITER $Q_{\mathrm{DT}}=10$ reference regime $\left(n / n_{\mathrm{GW}} \sim\right.$ $\left.0.85, H_{98(y, 2)} \sim 1, \beta_{\mathrm{N}} \sim 1.8\right)$. ELMy H-mode plasmas have been realized reaching or even exceeding those parameters in steady-state conditions (up to $\sim 5 \mathrm{~s}$ or $12 \tau_{E}$ ) in a reproducible way and only limited by the duration of the additional heating phase. These results have been obtained (a) in highly triangular plasmas, by increasing the average 
triangularity $\delta$ towards the ITER reference value $(\delta \sim 0.5)$, and (b) in plasmas at low triangularity $(\delta \sim 0.2)$ by seeding of Ar and placing the X-point of the plasma on the top of the septum. Pellet injection from the high field side is a third method yielding high density and high confinement, albeit not yet under steady-state conditions. In highly triangular plasmas the influence of input power, plasma triangularity and impurity seeding with noble gases has been studied. Density profile peaking at high densities has been obtained in (a) impurity seeded low triangularity discharges, (b) ELMy H-modes with low levels of input power and (c) discharges fuelled with pellet injection from the high field side. New ELM behaviour has been observed in high triangularity discharges at high density, opening a possible route to ELM heat load mitigation, which can be further amplified by Ar impurity seeding. Current extrapolations of the ELM heat load to ITER show possibly a window for Type IELM operation. Confinement scaling studies indicate an increase in confinement with triangularity and density peaking, and a decrease in confinement with the Greenwald number. In addition, experiments in $\mathrm{H}$ isotope and He indicate $\tau_{E} \propto M^{0.19} Z^{-0.59}$. The threshold power for the $\mathrm{L}-\mathrm{H}$ transition in He plasmas shows the same parametric dependence as in D plasmas, but with a 50\% higher absolute value.

PACS numbers: 52.55.Fa, 52.55.s

\section{Introduction}

The reference scenario for the ITER $Q=10$ scenario is the ELMy H-mode with density $n / n_{\mathrm{GW}}=0.85$ (where $n_{\mathrm{GW}}$ is the Greenwald density [1] given by $n_{\mathrm{GW}}\left[10^{20} \mathrm{~m}^{-3}\right]=$ $I_{\mathrm{p}}[\mathrm{MA}] /\left(\pi a^{2}[\mathrm{~m}]\right)$, where $I_{\mathrm{p}}$ is the plasma current and $a$ the minor radius of the plasma), energy confinement $H_{98(y, 2)} \sim 1$ (where $H_{98(y, 2)}$ is the confinement enhancement factor as given by the $\operatorname{IPB}(98(y, 2))$ scaling law [2]) and normalized beta value $\beta_{\mathrm{N}} \sim 1.8$ (with $\beta_{\mathrm{N}}=\beta_{\mathrm{t}} a[\mathrm{~m}] B_{\mathrm{t}}[\mathrm{T}] / I_{\mathrm{p}}[\mathrm{MA}]$ and $B_{\mathrm{t}}$ the toroidal magnetic field on axis). A common feature of ELMy H-mode plasmas is the degradation of confinement with increasing densities, rendering the simultaneous realization of high density and high confinement in such plasmas difficult. Over the past two years however we have shown that it is possible on JET to realize the normalized density and confinement parameters for ITER simultaneously with three different methods: (a) by increasing the average plasma triangularity $\delta$ to values close to those projected for ITER (section 2), (b) by extrinsic impurity seeding in low and high triangularity plasmas (section 3) and (c) with an optimized pellet injection sequence from the high field side in medium $\delta$ plasmas (section 4). Peaked plasma density profiles have been obtained in steady-state conditions in JET with careful tuning of the gas fuelling and plasma heating (section 5), with impurity seeding and with pellet injection from the high field side, although not yet under stationary conditions. Attention has also been paid to the mitigation of the high divertor power loads that can result during ELMs. Two promising routes, discussed in section 6, are being explored: (a) high density high triangularity plasmas leading to reduced ELM losses, lower frequency ELMs, and indications for Type II ELMs and (b) seeding of impurities creating a radiating mantle around the plasma. New results on the scaling of the heat load due to ELMs indicate a possibility for Type I operation in ITER. Confinement and $\mathrm{L}-\mathrm{H}$ power threshold scalings have been refined using the new results and with data from He plasmas in JET (section 7).

\section{High density and high confinement in highly triangular plasmas}

Experiments in JET, DIII-D, JT60-U and ASDEX [3-6] have shown the beneficial effect of increasing triangularity to reach high density and high confinement. This study has now been extended on JET [7], by increasing the average triangularity of the plasmas to values $\delta=0.47$, close to the ITER specifications. The time evolution of the main plasma parameters of a high density plasma at $I_{\mathrm{p}} / B_{\mathrm{t}}=$ $2.5 \mathrm{MA} / 2.7 \mathrm{~T}$ is given in figure 1 . High $\delta$ discharges reach high density $\left(n / n_{\mathrm{GW}} \sim 1.1, n \sim 1 \times 10^{20} \mathrm{~m}^{-3}\right)$ and confinement $\left(H_{98(y, 2)} \geqslant 1, \beta_{\mathrm{N}}=1.8-2\right)$, exceeding what is required for the $Q=10$ ELMy H-mode operation in ITER, simultaneously for a duration of about 4-5 s (or 9-11 energy confinement times), limited only by the length of the heating pulse. Increased confinement is not due to density peaking as plasma density profiles show only a moderate peaking with $n / n_{\text {ped }} \sim 1.2$ (with $n_{\text {ped }}$ and $n$, respectively, the density measured by the interferometer channel at $R=3.75 \mathrm{~m}$ and $R=3.02 \mathrm{~m}$ in JET). Figure 2 shows the evolution of the confinement enhancement factor $H_{98(y, 2)}$ versus the Greenwald fraction $n / n_{\mathrm{GW}}$ for different triangularities studied in JET. Data at the highest triangularities have been obtained in two configurations: (i) one matching very closely the projected

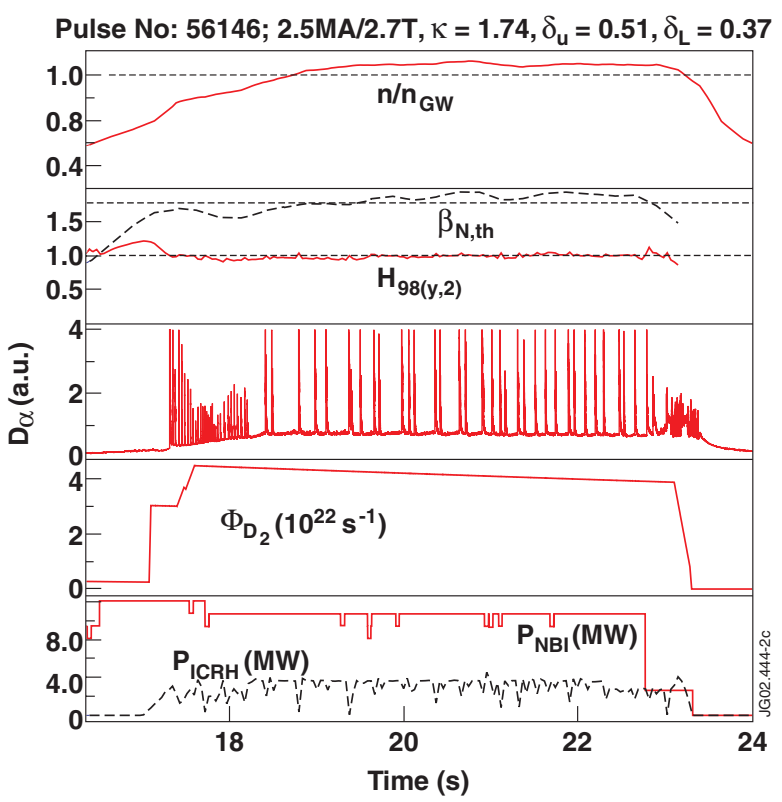

Figure 1. Discharge at high $\delta$ reaching simultaneously high confinement, beta and density for about $5 \mathrm{~s}$. 


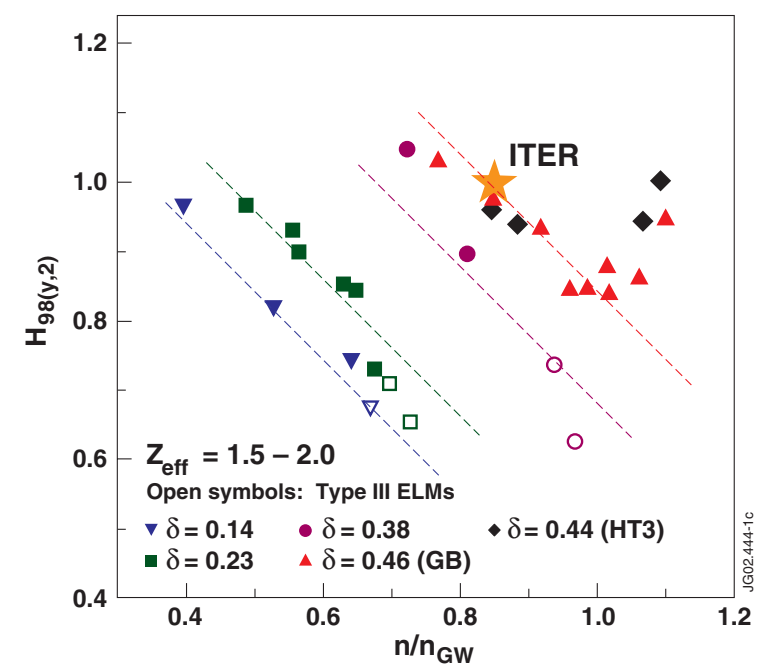

Figure 2. $H_{98(y, 2)}$ versus Greenwald factor under stationary conditions for various plasma triangularities.

ITER configuration in the Gas Box Divertor (upwards pointing triangles), and (ii) in a configuration designed to minimize the vertical forces on the tokamak vessel in the event of a disruption (HT3 configuration, diamonds) keeping similar average $\delta$ and $\kappa$ as the ITER configuration. This latter configuration is suited for operation at higher plasma currents (up to 3.5 MA, possibly $4 \mathrm{MA})$. Data points used for this graph are from discharges heated with NBI heating only, except for the HT3 ones, which were heated by NBI with up to $\sim 20 \%$ of the total input power provided by ICRH. The enhancement factor $H_{98(y, 2)}$ degrades with increasing density for all triangularities. The best discharges at present are reached at high triangularity and reach simultaneously $n / n_{\mathrm{GW}}=1.1$ and $H_{98(y, 2)} \geqslant 1$. (Note that the confinement data shown here are obtained using an improved equilibrium calculation in JET [8] with respect to earlier publications, but the essential conclusions remain unchanged.) Note, however, that discharges at low $\delta$ can reach similar high performance with pellet injection or impurity seeding (in discharges where the X-point is located on the top of the septum), as discussed, respectively, in sections 3 and 5. Good confinement and density conditions are observed in the presence of Type I ELMs with a reduced ELM size and frequency. New and interesting ELM behaviour has been observed in these high triangularity discharges at high density, opening a new route for ELM mitigation. This will be further discussed in section 6. At the highest densities, beam deposition profiles become flat or even hollow. Rather stiff electron profiles but less stiff ion temperature profiles are observed. The beneficial effects of high triangularity in reaching high density and confinement in JET could possibly be linked to improved access of the plasma edge to second stability, as seen on other tokamaks, e.g. DIII-D [9]. A tradeoff has been observed between triangularity and heating power to reach high density and high confinement. At medium triangularities $(\delta \sim 0.30)$ higher heating powers $P_{\mathrm{IN}} / P_{\mathrm{L}-\mathrm{H}}>$ 2.6 are needed to obtain the same results at $\delta \sim 0.5$ where $P_{\mathrm{IN}} / P_{\mathrm{L}-\mathrm{H}} \sim 1.4$. The condition for sufficient heating power at a given $\delta$ in order to obtain high confinement is correlated with maintaining the pedestal temperature above the critical temperature for Type III ELMs. Common to the high $\delta$ experiments at low $P_{\mathrm{IN}} / P_{\mathrm{L}-\mathrm{H}}$ and the low $\delta$ ones at high $P_{\mathrm{IN}} / P_{\mathrm{L}-\mathrm{H}}$ is the observation of a higher pedestal density $n_{\mathrm{e}, \text { ped }}$ for a given edge temperature $T_{\mathrm{e}, \text { ped }}$. Instead of the usual decrease of $T_{\mathrm{e} \text {,ped }}$ with increasing $n_{\mathrm{e} \text {,ped }}\left(T_{\mathrm{e} \text {, ped }} \propto 1 /\left(n_{\mathrm{e}, \mathrm{ped}}\right)^{2}\right)$, at densities $n_{\mathrm{e} \text {,ped }} \geqslant 0.75 n_{\mathrm{GW}}$, the pedestal temperature $T_{\mathrm{e} \text {,ped }}$ remains almost constant and above the critical temperature for reversion to Type III ELMs [10]. Higher plasma triangularities are beneficial to realize this condition, due to the increased edge stability, and could explain why lower values of $P_{\mathrm{IN}} / P_{\mathrm{L}-\mathrm{H}} \sim$ 1.4 are sufficient to maintain high performance.

\section{Impurity seeding in low and high triangularity plasmas}

Impurity seeding has been successfully applied in TEXTOR, DIII-D, JET and JT-60U over the past years [11] leading not only to high levels of radiation in the edge but also to favourable regimes with enhanced confinement. Work on impurity seeding, started on JET in 1999 [12], has been continued and extended over the past two years in plasmas with low and high triangularity [13-17]. In low triangularity plasmas $(\delta \sim 0.2)$, the $\mathrm{X}$-point has been lowered onto the top of the septum, which then plays effectively the role of a limiter. This does not lead to a loss of the H-mode, but on the contrary, easier access to the H-mode is obtained as the $\mathrm{L}-\mathrm{H}$ power threshold is reduced under such conditions [18], facilitating $\mathrm{H}$-mode operation at high radiation fractions. The scenario consists of a 'puff' and 'afterpuff' phase as detailed in [13]. Values in the afterpuff phase reach $n / n_{\mathrm{GW}} \sim 0.85$ with confinement $H_{98(y, 2)} \sim 1$, representing a significant enhancement in confinement compared to what is otherwise obtained in low triangularity plasmas, for periods of about $5 \mathrm{~s}$ or $12 \tau_{E}$, only limited by the duration of the heating pulse. For a given temperature, higher densities are reached with impurity seeding in the afterpuff phase with peaking factors up to $n(0) / n_{\text {ped }} \sim 1.8$.

Impurity seeding has also been applied to plasmas with high triangularity (configurations similar to the ones of section 2) with the X-point well above the dome of the septum. As confinement in these plasmas is less sensitive to strong gas puffing as indicated in section 2, the scenario used in this case does not consist of a 'puff' and 'afterpuff' phase, but a constant deuterium gas puff is applied (figure 3), together with a careful dosing of impurities. The figure shows clearly the integrated nature of these discharges when Ar is present $(t>20 \mathrm{~s}$ ): (i) high radiation: increased radiation (up to $70 \%$ ) with formation of a radiating belt (see figure 4), (ii) high confinement $\left(H_{98(y, 2)} \sim 0.9\right)$ and thermal $\beta_{\mathrm{N}} \sim 2.1$ (without change in the stored energy of the plasma during Ar seeding) and (iii) high density (up to $n / n_{\mathrm{GW}}=1.2$ ), linked to an increase of the product $f \times \tau_{\mathrm{p}}$, where $f$ is the fuelling efficiency and $\tau_{\mathrm{p}}$ the particle confinement time. The collapse at the end of the discharge is due to a lack of central NBI heating at the very high densities reached. IR measurements in the inner and outer divertors show a significant reduction of the power flux to the divertor target plates and therefore of their surface temperature [15]. Probe measurements [15] reveal reduced electron temperatures in front of the target plates, corresponding to some degree of detachment. In addition, the ELM frequency decreases with 


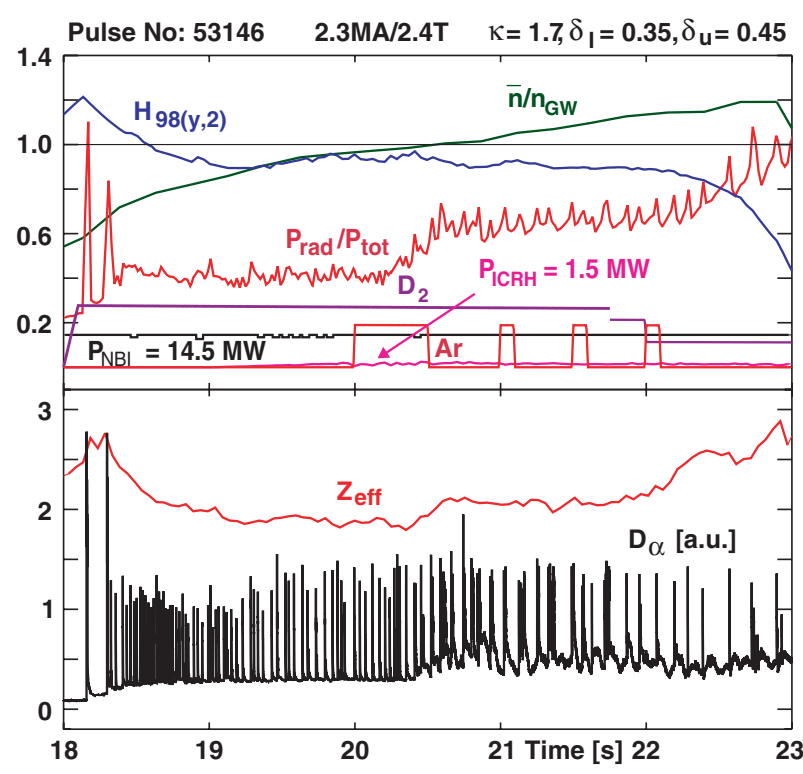

Figure 3. Discharge with Ar seeding at high $\delta$ reaching simultaneously high confinement, beta and density for about $5 \mathrm{~s}$.

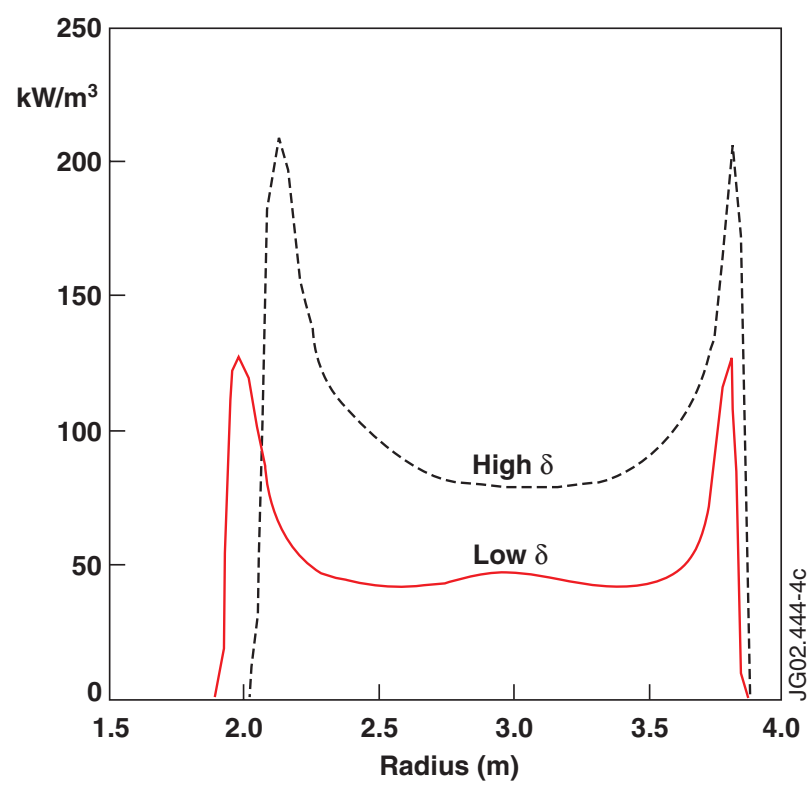

Figure 4. Radiation profiles obtained with Ar seeding in high (\#53146) and low (\#50473) triangularity plasmas.

increasing radiation, consistent with the observation that the ELM frequency is roughly proportional to the difference between deposited heating power and radiated power. Note in particular that the change in the character of the ELMs with Ar seeding points to a modification in pedestal parameters, but the global confinement has not changed (see discussion in section 6.3). For given plasma conditions impurity seeding is thus an additional tool to reduce the detrimental influence of the ELMs, without reduction of confinement. The best conditions are realized when pumping is reduced (and thus recycling is more pronounced). Clear radiating mantles for the septum (low $\delta$ ) and ITER-like (high $\delta$ ) cases are obtained, as illustrated in figure 4 [19]. Calculations with TRANSP show lower energy transport in the core [11]; in addition,

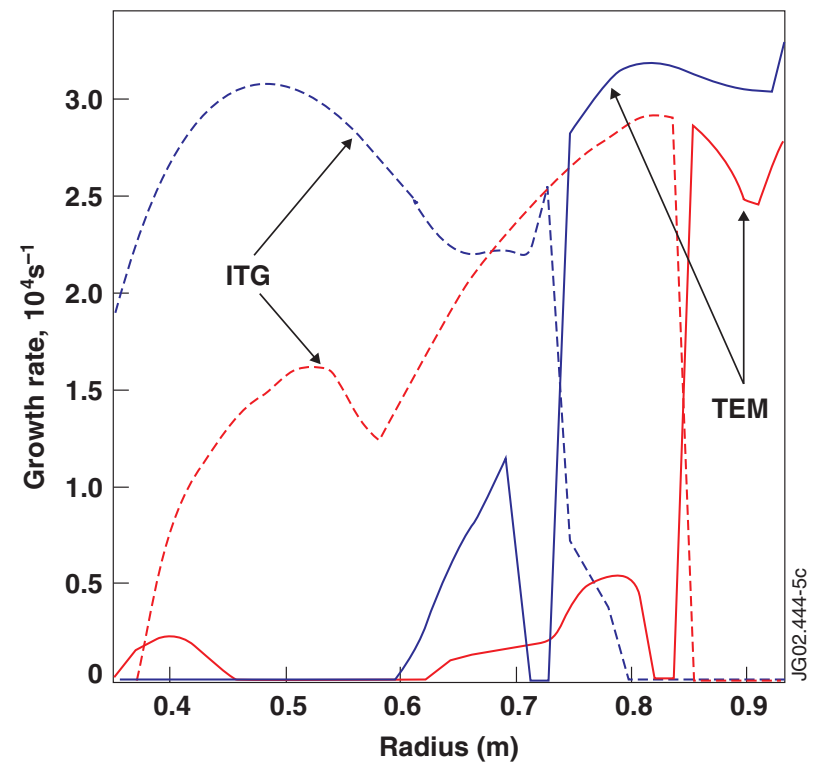

Figure 5. Growth rates of ITG modes (- - - ) and TEM modes (- ) for the high $\delta$ discharge of figure 3 (lower traces) with $\mathrm{Ar}$ seeding. Compared to a reference discharge without Ar seeding (upper traces) the growth rate of the ITG instability is strongly reduced in the inner part of the plasma.

simulations with the multi-mode model [20] implemented in ASTRA [21] and JETTO [22] indicate a clear reduction of the ITG growth rate in both high and low $\delta$ discharges (figure 5). This is due to an increased dilution in the radiating zone, whereas the dilution in the centre remains unchanged [23]. Analogous confinement improvement has been found due to impurity seeding in L-mode discharges in DIII-D and JET [24]. Application of central ICRH is beneficial to avoid central impurity accumulation [17]. Depending upon the details of Ar seeding, impurity accumulation can be observed, generally correlated in time with sawtooth suppression and peaking of the impurity profile in the centre. The sawtooth suppression is linked to an increase in the value of the central $q$ above unity. Application of on-axis ICRH heating prevents the $q(0)$ value from rising above unity, permitting the high performance phase to be kept for the whole heating period.

\section{Optimized pellet sequence to reach high density with high confinement}

The potential of high field side pellet injection for fuelling the plasma to high densities with simultaneous high confinement has been investigated in ELMy H-mode discharges in JET [25]. An optimized fuel cycle can avoid too excessive prompt particle losses leading to an increase in the neutral gas pressure and edge density. The optimization strategy consists in a first phase where the density is ramped up (while allowing for a small confinement loss) followed by a second phase minimizing the particle flux in order to maintain the density and to recover confinement. This is shown in figure 6. As a target, a plasma was chosen at $I_{\mathrm{p}} / B_{\mathrm{t}}=2.5 \mathrm{MA} / 2.4 \mathrm{~T}$ with $\delta \approx 0.34$ showing a confinement collapse when trying to raise the density beyond $n / n_{\mathrm{GW}}=0.8$ with strong gas puffing. In such a plasma solid $4 \mathrm{~mm}^{3}$ deuterium cubes were launched 


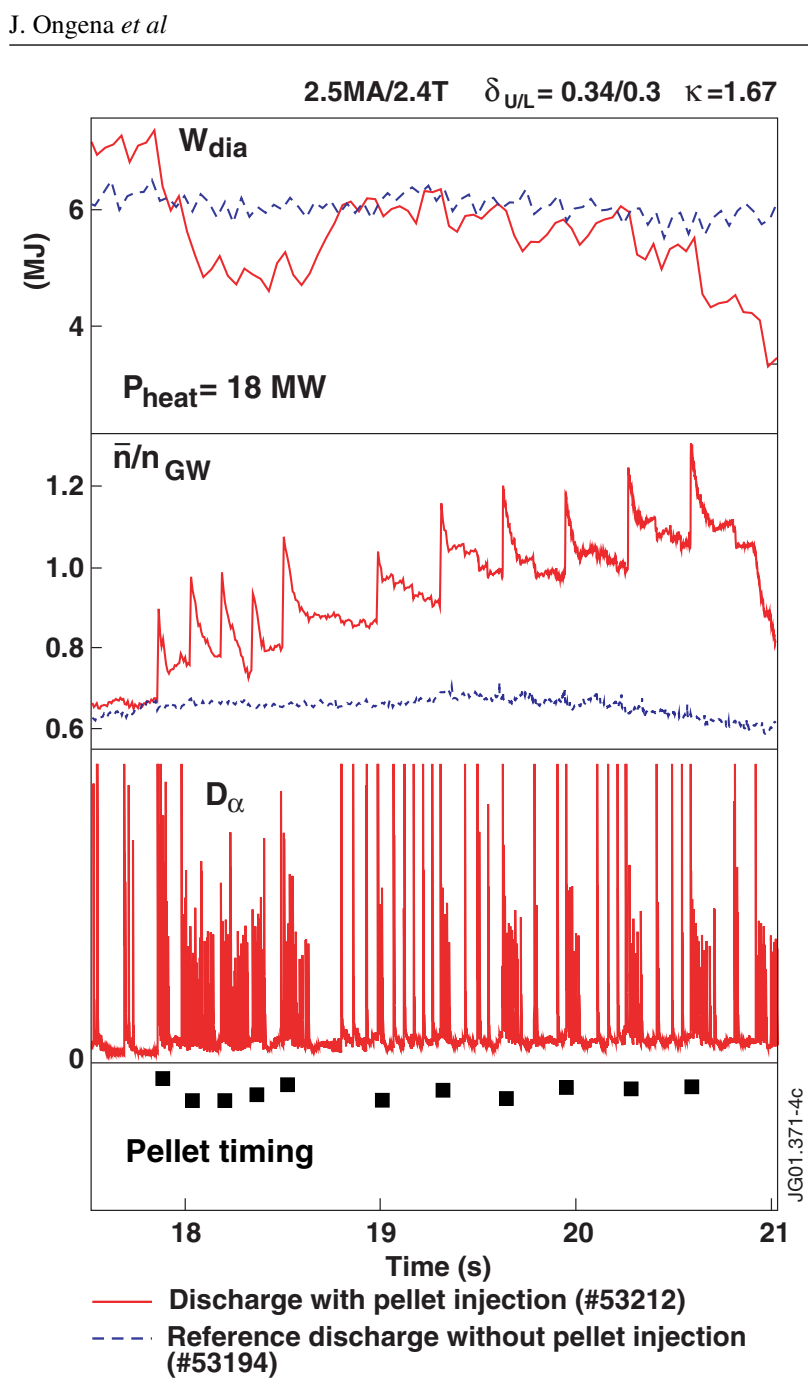

Figure 6. Example of a discharge with HFS pellet injection with an optimized fuel cycle. Dashed lines correspond to a reference discharge without pellet injection or gas puff.

at a speed of $160 \mathrm{~m} \mathrm{~s}^{-1}$ from the high field side downward at an angle of $44^{\circ}$ with respect to the horizontal plane and with a tangency radius at a normalized minor radius $\rho \sim 0.6-0.7$. The optimization strategy translated into launching a first pellet string at $6 \mathrm{~Hz}$ to raise the density, followed by a pellet string at $2 \mathrm{~Hz}$, which is sufficiently slow to allow confinement recovery after each pellet. In this way the plasma density increased until finally a density above the Greenwald limit is achieved with simultaneous high plasma energy content of about $6 \mathrm{MJ}$ (corresponding to a $H_{98(y, 2)} \sim 0.82$ ) with $\beta_{\mathrm{N}}>1.8$. Plasma density profiles show large peaking factors $n(0) / n_{\text {ped }} \approx 2$, accompanied by an almost adiabatic reduction of the electron temperature. $Z_{\text {eff }}$ decreased to about 1.7 at the highest densities reached.

Transport simulations of pellet fuelled discharges have been performed with the JETTO code using both the semiempirical mixed Bohm/gyro-Bohm model [22] and the physics-based Weiland model [26]. It is seen that in order to reproduce the density profile evolution the edge diffusion after pellet injection has to be increased by a factor of 3-4. Moreover, to reproduce the profile peaking an anomalous convective flux has to be introduced in the simulation. This is provided by an anomalous pinch velocity $v=0.25$ $\times D \operatorname{grad}(q) / q$ in the mixed Bohm/gyro-Bohm model and by the effect of the off-diagonal elements of the transport matrix in the Weiland model. In all the simulations, a dramatic improvement of the core particle confinement in comparison with the pre-pellet phase is not needed in order to explain the profile evolution.

\section{Long timescale density peaking}

Long timescale density peaking has been observed in JET plasmas at densities exceeding the Greenwald value [27]. These neutral beam heated discharges are characterized by Type I ELMs and good energy confinement. A power scan in $1.9 \mathrm{MA} / 2.0 \mathrm{~T}$ plasmas shows that at low NBI heating powers $\left(P_{\mathrm{NBI}}<8 \mathrm{MW}\right)$, the plasmas obtained are transient, with a density profile peaking increasing linearly with time, reaching values up to $n(0) / n_{\text {ped }} \sim 2$. The central plasma temperature drops, the current profile broadens and sawteeth are lost, followed by a radiative collapse of the plasma centre. At high NBI heating powers $\left(P_{\mathrm{NBI}}>12 \mathrm{MW}\right)$, the central density is limited by higher $m / n=4 / 3$ neoclassical tearing modes (NTMs). These limitations can be avoided at intermediate NBI heating levels $\left(P_{\mathrm{NBI}} \sim 10 \mathrm{MW}\right)$, sometimes assisted by low power $\left(P_{\mathrm{ICRH}}=1-2 \mathrm{MW}\right)$ centrally deposited ICRH (to mitigate a radiative collapse of the centre), and with an optimized gas waveform to reduce the size of the first ELM, to avoid triggering a large $m / n=3 / 2$ NTM, detrimental to confinement. A gas position scan showed that gas puffing from the inner ring into the private divertor region is beneficial for density peaking. Data for a plasma obtained under such conditions are summarized in figure 7 . In this plasma, sawteeth are preserved until the end of the heating phase, at a line-averaged density $n / n_{\mathrm{GW}}=1$ and with a density peaking $n(0) / n_{\text {ped }} \sim 1.3$, at high confinement $H_{98(y, 2)} \sim 0.96$ and $\beta_{\mathrm{N}}=2$. In discharges with dominant ICRH $\left(P_{\mathrm{NBI}} / P_{\mathrm{ICRH}}<0.25\right)$, the density profile was not peaked. However, the conditions at the edge (ELM frequency and size, recycling) were different to those in plasmas heated with NBI only, possibly explaining the difference in density peaking behaviour. Analysis of the discharge in figure 7 at $\rho \sim 0.4$ using the JETTO and TRANSP codes shows that the density peaking observed can be explained by the Ware pinch with the ratio of particle to heat diffusivity of $D / \chi_{\text {eff }} \sim 0.25-0.4$. In that case, NBI particle fuelling and Ware pinch contribute equally to the density peaking.

\section{ELM mitigation studies}

The loss of power from the plasma due to ELMs is given by $P_{\mathrm{ELM}}=f_{\mathrm{ELM}} \times \Delta W_{\mathrm{ELM}}$, where $f_{\mathrm{ELM}}$ is the ELM frequency, determined by edge transport and the power through the separatrix and $\Delta W_{\mathrm{ELM}}$ is the energy lost with each ELM, depending on the plasma edge parameters. Tools to influence both parameters are triangularity, D puffing rate, Ar seeding and additional heating power level. Figure 8 shows the evolution of the ratio $P_{\mathrm{ELM}} / P_{\mathrm{IN}}$ as a function of the D puffing rate, where $P_{\mathrm{IN}}$ is the total power input to the plasma. At low or medium $\delta$ the ratio $P_{\mathrm{ELM}} / P_{\mathrm{IN}}$ is accompanied at high fuelling rates with a transition from Type I to Type III ELMs. 

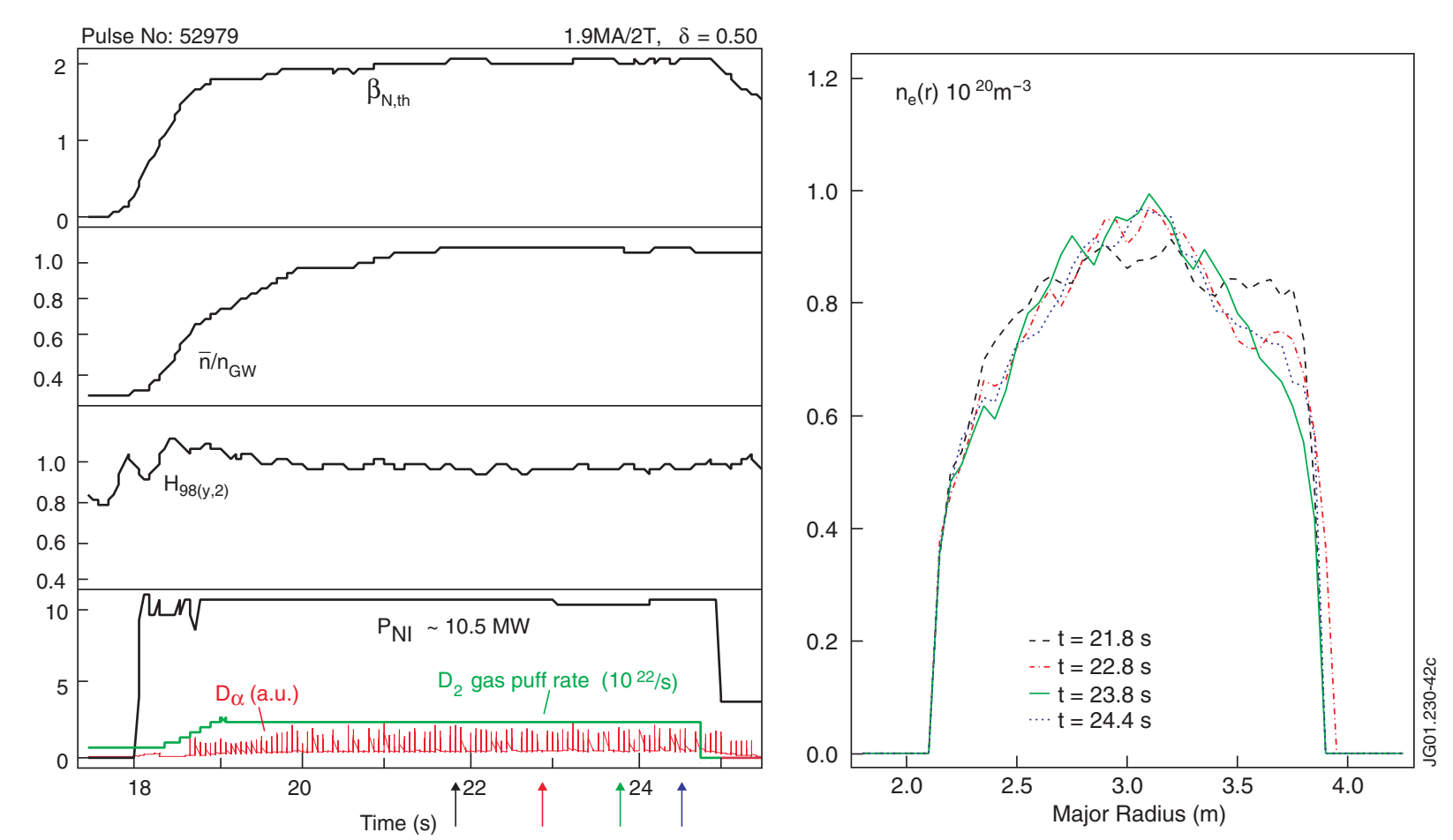

Figure 7. Example of a discharge with spontaneous and stationary density peaking. Density profiles are shown for the times indicated by the arrows.

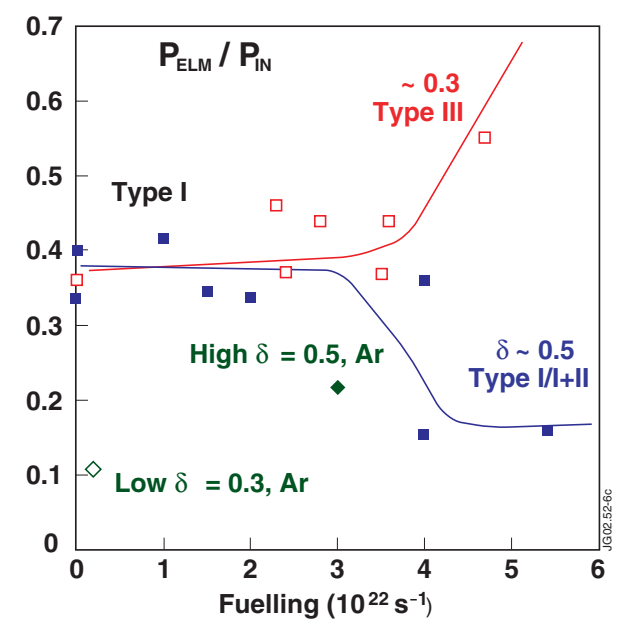

Figure 8. Average power loss fraction due to ELMs as a function of D puff rate.

In contrast, at high $\delta$, the ratio $P_{\mathrm{ELM}} / P_{\mathrm{IN}}$ decreases at high fuelling rate and Type I ELMs are kept, with indications for additional Type II like activity, correlated with broadband interELM MHD activity (as is also seen on other machines [31,32]). In figure 8 in addition the influence of Ar seeding can be seen at both high and low $\delta$, showing that in both cases $P_{\mathrm{ELM}} / P_{\mathrm{IN}}$ is further reduced [34].

6.1. Observation of reduced ELM losses at high density and high confinement in high triangularity plasmas without impurity seeding

The decrease of $P_{\mathrm{ELM}} / P_{\mathrm{IN}}$ at high density is due to the fact that with increasing D puffing the Type I ELM frequency first increases, then reaches a maximum to decline again $[28,29]$. This new ELM behaviour breaks the usual link between $f_{\mathrm{ELM}}$ and $W_{\mathrm{ELM}}$, observed at low $\delta$ and lower input powers [30]. Under such conditions, reduced ELM losses are observed and a new loss mechanism is seen in between ELMs together with the appearance of mixed Type I/II ELMs $[7,28]$. With increasing densities, the pedestal pressure does not decrease further, mainly because the pedestal temperature remains constant with increasing density. Consequently, high values for $H_{98(y, 2)} \sim 1$ and density $n / n_{\mathrm{GW}}>0.9$ are realized simultaneously, as shown in section 2. The MHD appears as an increased level of density and broadband $(15-50 \mathrm{kHz})$ magnetic fluctuations at high poloidal to toroidal periodicity, suggesting edge localization [28], reminiscent of 'quasicoherent' modes associated with EDA modes in Alcator-C [31] and Type II ELMs in ASDEX-Upgrade [32]. The reduction in $\Delta W_{\text {ELM }} / W_{\text {ped }}$ (with $\Delta W_{\text {ELM }}=W_{\text {dia, before ELM }}-W_{\text {dia, after ELM }}$ and $W_{\text {ped }}=\frac{3}{2}\left(n_{\mathrm{e} \text {,ped }}\left(T_{\mathrm{e} \text {,ped }}+T_{\mathrm{i} \text {,ped }}\right)\right) V_{\mathrm{p}}$, where the pedestal values are taken at the top of the $\mathrm{H}$-mode pedestal just before the ELM and $V_{\mathrm{p}}$ is the confined plasma volume) is nearly entirely due to the decrease of $\Delta T_{\mathrm{e} \text {,ped }} / T_{\mathrm{e} \text {,ped }}$ with increasing density (see figure 9). In discharges with $\Delta T_{\text {e,ped }} / T_{\text {e,ped }} \sim 0$ the drop in pedestal pressure due to the ELM is entirely due to the (small) change in pedestal density (figure 9). A value of $\Delta T_{\mathrm{e} \text {,ped }} / T_{\mathrm{e} \text {,ped }}=0$ is obtained in plasmas with high upper triangularity $\left(\delta_{\mathrm{u}} \sim 0.5\right)$ and reduced lower triangularity $\left(\delta_{1} \sim 0.3\right)$, leading to so-called 'minimum' ELMs, with $\Delta W_{\mathrm{ELM}} / W_{\text {ped }} \sim 4 \%$ (at $H_{98(y, 2)} \sim 1.05$ and $n / n_{\mathrm{GW}} \sim 0.8$ ), the lowest value obtained so far on JET, and very well within the range required for ITER $\left(3 \%<\Delta W_{\mathrm{ELM}} / W_{\text {ped }}<15 \%\right)$. 


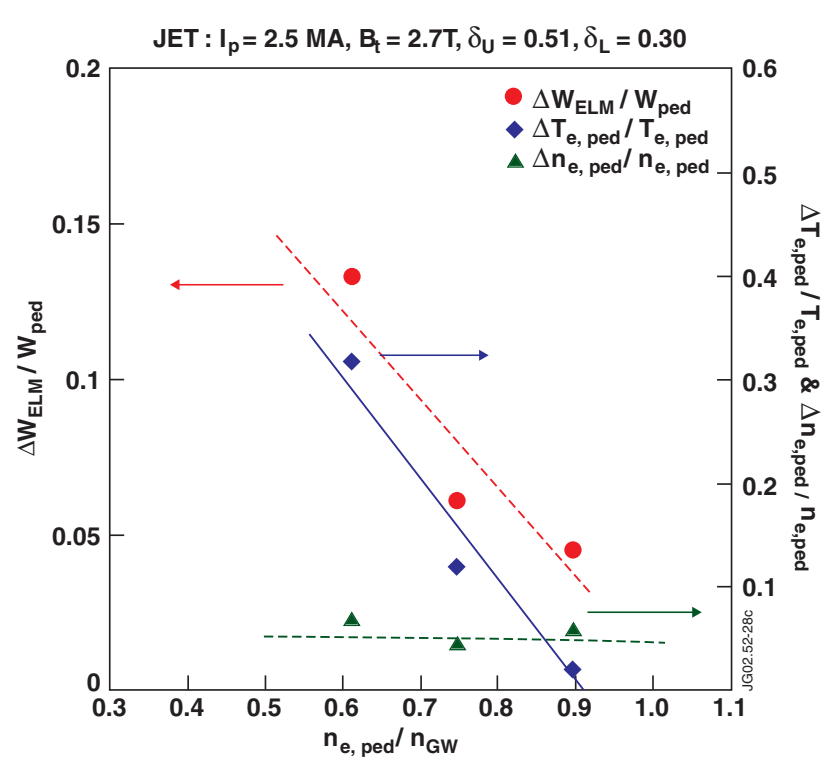

Figure 9. ELM size, temperature and density change during the ELM as a function of pedestal density.

\subsection{ELM mitigation by impurity seeding}

For both low and high $\delta$ discharges studied, the increase in radiated power due to $\mathrm{Ar}$ seeding leads to reduced target loads, with only a minor influence on $Z_{\text {eff }}$ (typically $\Delta Z_{\text {eff }} \sim 0.2-0.3$ ), with an increase in density for a given confinement quality (see section 3 ).

6.2.1. Low or medium $\delta$ discharges. In the afterpuff of low $\delta$ septum discharges, the lower $\mathrm{D}_{2}$ puffing rate leads usually to a reduced frequency and Type I ELMs. The addition of Ar further reduces the ELM frequency. Not only is the frequency of the ELM reduced with Ar seeding, but there is also a reduction of $\Delta W_{\mathrm{ELM}} / W_{\text {ped }}$ correlated with an increase of edge electron collisionality or ion parallel transit time $\tau_{\| \text {front }}$ (see section 6.3). A significant divertor surface temperature reduction due to $\mathrm{Ar}$ seeding has been measured by IR thermography of the inner and outer target plates in recent experiments at medium $\delta$ [33] (in the so-called DOC configuration especially developed to allow detailed thermographic measurements) as shown in figure 10, where data are compared from discharges with roughly the same confinement and core parameters, with and without impurity seeding. Note that not only during the ELM but in particular also in between the ELMs the surface temperature is reduced. This unique feature of $\mathrm{Ar}$ seeding will allow for larger temperature excursions due to ELMs without reaching the ablation limit of the target material.

6.2.2. High $\delta$ discharges. A radiating belt is formed (figure 4) associated with a significant increase in the radiated power fraction. In addition, Ar seeding also causes a drop in ELM frequency, together with an increase in the base level $D_{\alpha}$ emission, indicative of increased recycling between ELMs, resembling the phenomena seen with mixed Type I/II ELMs in unseeded high $\delta$ discharges. The ELM frequency is reduced compared with the unseeded reference (see figure 3 ), with almost constant $\Delta W_{\mathrm{ELM}} / W_{\text {ped }}=5-7 \%$ [15] leading to a reduced divertor load averaged over the ELMs. The strong moderation is also supported by IR heat flux measurements (as in the low $\delta$ case) and the marked drop of the electron temperature just in front of the target plates measured with Langmuir probes [15]. A partial detachment of the plasma in the divertor is seen under those conditions. Similar ELM moderation as with Ar seeding can be obtained with strong D puff, but (i) without the beneficial effects of the increase in particle confinement time (leading to a higher density for the same high energy confinement), and (ii) without the formation of a radiating belt, decreasing the overall heat flux towards the target plates.

\subsection{ELM characteristics and plasma parameters}

A strong correlation has been found between the Type I ELM size and either the dimensionless electron collisionality at the top of the pedestal or the transit time of the ELM heat front to the target plate (calculated with pedestal parameters) $\tau_{\|}^{\text {front }}[34]$ for all plasma conditions studied, including impurity seeding and the new Type I ELM behaviour described above (see figures $11(a)$ and $(b)$ ). JET has thus shown that the pedestal parameters determine the ELM size, the frequency being determined for a given power flux through the separatrix by the transport in between ELMs in the plasma edge region $[28,29]$. The beneficial reduction in ELM size at high gas puffing rates in both high $\delta$ plasmas, and plasmas with impurity seeding can thus be understood as due to the resulting increase of edge collisionality or the increase of $\tau_{\|}^{\text {front }}$. Figures $11(a)$ and (b) show the present estimate of the acceptable ELM energy in ITER based on divertor lifetime $(<0.01 \mu \mathrm{m}$ erosion per ELM) for both experimental correlations and for two assumptions on the ELM energy loss from the bulk plasma that reaches the divertor target. The upper and lower values in these ranges originate from differences in the assumptions on the detail of the ELM power deposition and of the angle of incidence of the field line on the ITER divertor target [35]. In order to determine whether the observed Type I ELMs are extrapolable to next step devices requires not only knowledge of the bulk plasma energy loss, but also detailed information on the characteristics of the ELM energy flux to the divertor target (proportion of the energy that reaches the divertor target, area over which is deposited, time, etc). In this respect, experiments with ELM time-resolved divertor energy flux have contributed to firm up the basis for the extrapolation of the present results to ITER. The ELM rise time $\tau_{\mathrm{IR}}$, as seen at the target by the fast IR camera, is proportional and larger than $\tau_{\|}^{\text {front }}$ by a factor 2-3 over a large range of plasma conditions for JET, ASDEX-Upgrade and JT-60U $[36,37]$. This has increased the duration of the expected power pulse in ITER from 220 to $500 \mu \mathrm{s}$ and therefore the maximum allowed ELM deposited energy by $\sim 1.5$ with respect to previous assumptions. The proportionality between $\tau_{\mathrm{IR}}$ and $\tau_{\|}^{\text {front }}$ observed for different machines gives credit to the scaling as a function of $\tau_{\|}^{\text {front }}$ for the extrapolation of the divertor heat load for ITER. This scaling, as shown in figure 11(b), decreases the constraints on the target plates for ITER. Further reductions of these constraints can be expected from (i) the fact that a substantial amount of the ELM energy (up to a half) arrives at the target after the maximum 

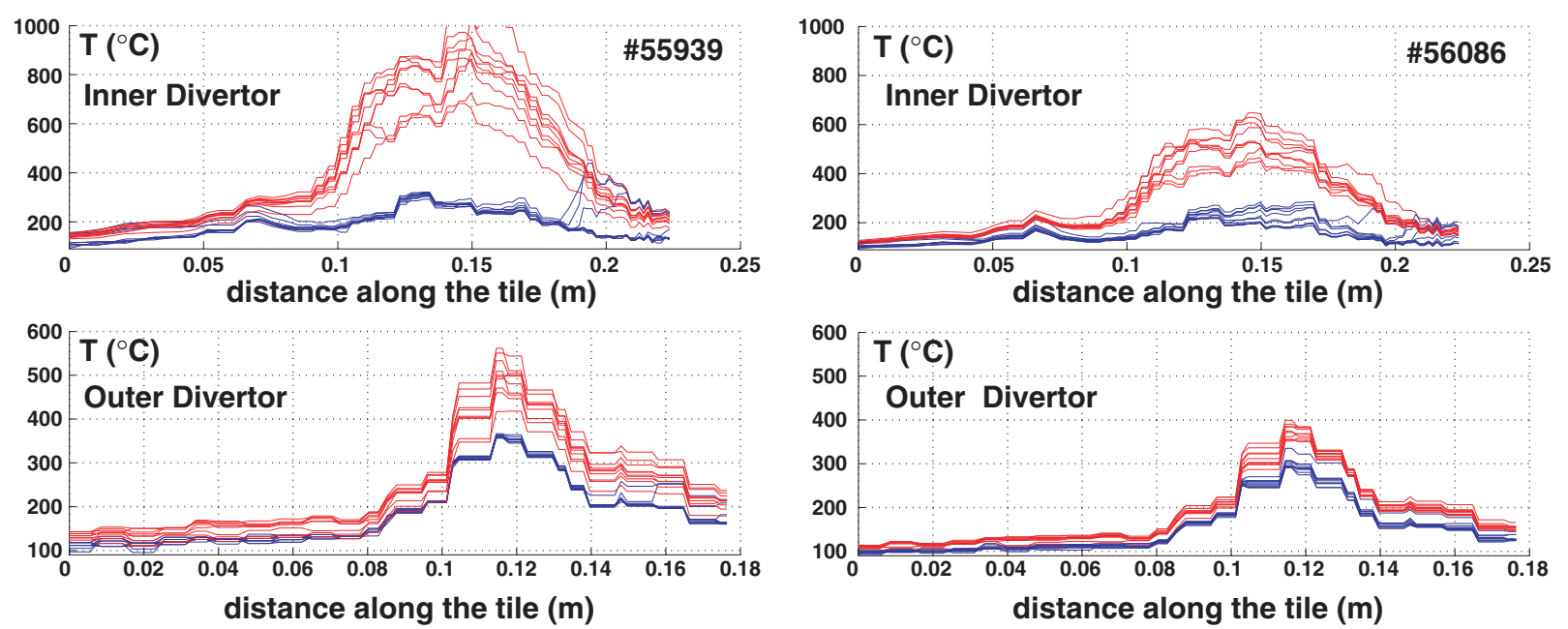

Figure 10. IR temperature profile as a function of the distance along the inner divertor target during (red) and in between (blue) ELMs for an unseeded (left) and Ar seeded (right) medium $\delta$ discharge. The different curves indicate the scattering in the measurement over the ELM cycle during which the data are taken.
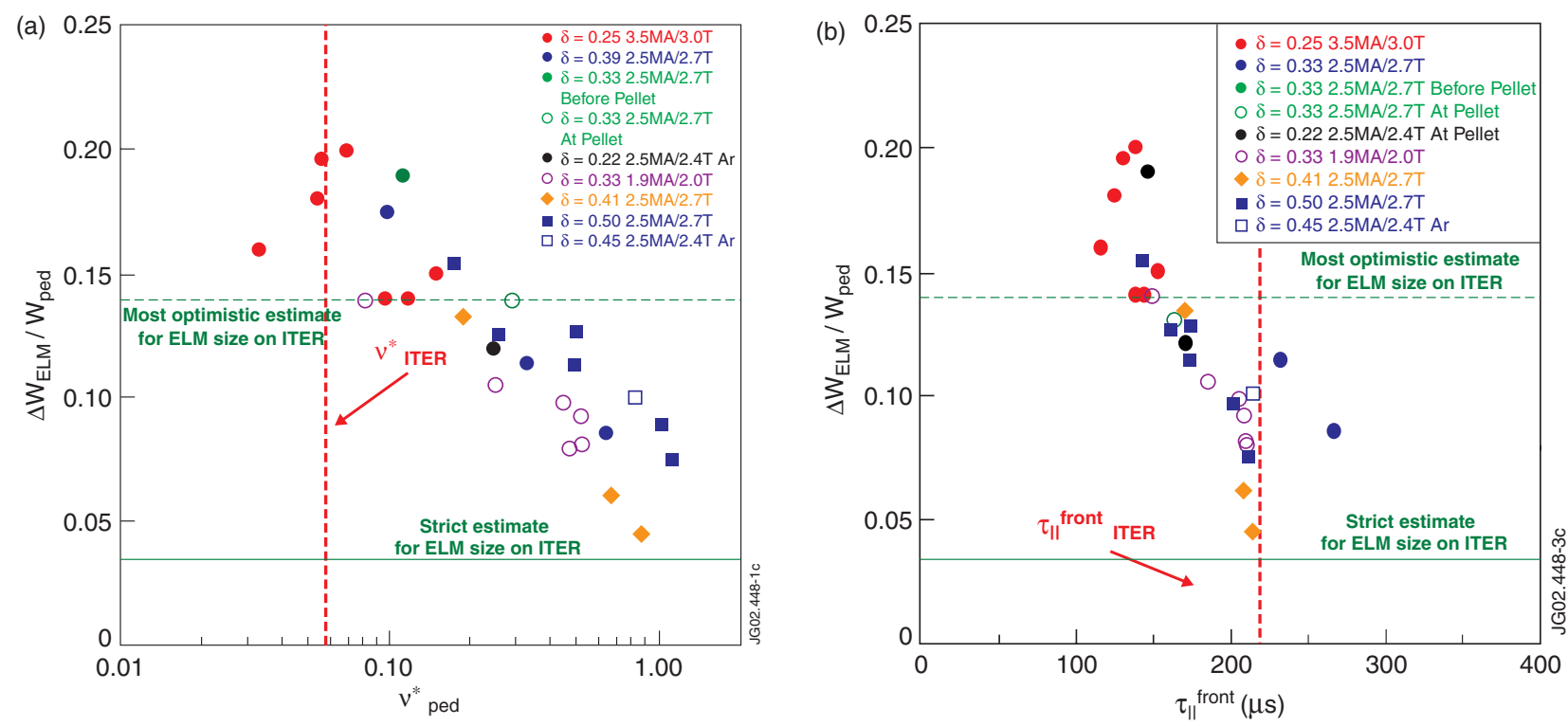

Figure 11. (a) ELM size versus neoclassical edge collisionality for a wide range of plasma conditions in JET. ELM size limits for ITER are indicated for (i) an inter-ELM load of $5 \mathrm{MW} \mathrm{m}^{-2}$ and an increased inclination of the divertor targets (- - - -) and (ii) for an inter-ELM load of $10 \mathrm{MW} \mathrm{m}^{-2}$ and the ITER reference design for the targets $(-)$. (b) Same data as $(a)$, but now plotted as a function of the transit time of the ELM heat front to the target plate $\tau_{\|}^{\text {front }}$ for a wide range of plasma conditions in JET. The same remarks apply as in $(a)$ to the indicated limits for the ELM size.

surface temperature has been reached [36,37], and (ii) that typically only $50-80 \%$ of the bulk plasma energy loss is found at the target plate. The above results indicate a possible window for operation of Type I ELMs in ITER, with an acceptable divertor lifetime.

\section{Confinement scaling and L-H power threshold studies}

Several confinement aspects have been studied at JET over the past three years. The new results obtained and discussed in sections 2-4 allowed the investigation of the influence of peaking, triangularity and proximity to the Greenwald limit on energy confinement [38], variables that are not contained in the current scaling expressions used to predict the performance of ITER. The best fit of the residuals of the ratio $\tau_{E, \exp } / \tau_{98(y, 2)}$ to a subset of the new data for which accurate pedestal values for $n_{\text {ped }}$ were available, was obtained using the variable $F_{\mathrm{q}}=$ $q_{95} / q_{\text {cyl }}[39]$ as a shaping parameter, and resulted in a corrected enhancement factor $H_{98(y, 2) \text {,corr }}=F \times H_{98(y, 2)}$, where $F=$ $0.46+1.35 \ln \left(F_{\mathrm{q}}\right)-0.17 n / n_{\mathrm{GW}}+0.38\left(n / n_{\mathrm{ped}}-1\right)$. The influence of these new parameters on ITER confinement is illustrated using ITER values for the density $n / n_{\mathrm{GW}}=0.85$ and shaping $F_{\mathrm{q}}=1.58$, with and without density peaking. This results in $H_{98(y, 2) \text {,corr }}=0.94$ without and $H_{98(y, 2) \text {,corr }}=$ 1.05 with a moderate peaking $\left(n / n_{\text {ped }} \sim 1.3\right)$, i.e. a $10 \%$ improvement. During the 2001 shutdown, the septum in the JET gasbox divertor has been removed, resulting in the 
'Septum Replacement Plate' or SRP divertor similar to the previous Mark II. A preliminary comparison of present data from the more open SRP divertor suggests a slight reduction in confinement, in line with comparable findings for Mark I and II [38]. A set of experiments in $\mathrm{He}$ (with a purity $C_{\mathrm{He}} / C_{\mathrm{D}}=85 \%$ ) [40] provided additional data for the isotope scaling of confinement. Combined with previous confinement data in $\mathrm{H}$ and $\mathrm{T}$ plasmas, a confinement scaling is found which depends on the mean mass $M$ and charge $Z$ of the bulk species as $\tau_{E} \propto M^{0.19} Z^{-0.59}$. The $\mathrm{L}-\mathrm{H}$ threshold power in He plasmas shows the same $B_{\mathrm{t}}, n_{\mathrm{e}}$ and mass dependence as for deuterium, but is $50 \%$ higher in absolute value.

\section{Conclusions and outlook}

The experimental campaigns of the last three years on JET have resulted in a simultaneous extension of density and confinement in ELMy $\mathrm{H}$-mode plasmas using various methods. By further increasing the average triangularity of the plasma, discharges have now been realized with values for $H_{98(y, 2)} \geqslant 1$ and $n / n_{\mathrm{GW}} \sim 1.1$, which exceeds what is required for the ITER $Q=10$ standard scenario. The flat-top duration of these discharges is about $5 \mathrm{~s}$ and is only limited by technical constraints on JET. These high density, high confinement discharges exhibit energy losses by other mechanisms than pure ELM activity, and this points to a possible way of ELM mitigation. High density in high $\delta$ discharges leads to a reduction in ELM frequency, which is in contrast to the usual relation between ELM frequency and ELM size. Detailed analysis of the ELM behaviour and additional thermographic measurements on the divertor targets indicate that there is a possible window for operation for Type I ELM discharges in ITER. Impurity seeding is an additional tool to mitigate ELMs in discharges with high and low triangularity and has no detrimental effect on energy confinement or neutron production. Pellet injection from the high field side with a tailored injection rate has also resulted in discharges with high density and high confinement, but more work is needed to optimize the stationarity. Discharges with strong density peaking $\left(n(0) / n_{\text {ped }} \sim 2\right)$ have also been obtained without pellet injection, by careful gas dosing over long time intervals. Density peaking and high $\beta$ values both favour the destabilization of NTMs due to the associated increased bootstrap current fraction. This can be a limiting factor for the confinement properties of such plasmas, unless care is taken to avoid the creation of large seed islands, induced by, e.g., large first ELMs or sawteeth, or by varying the ICRH phasing [41]. Confinement studies show an increase of energy confinement with density peaking and triangularity, but still a degradation of confinement with increasing Greenwald factor. Density peaking and plasma shaping have a beneficial influence on confinement. Confinement scaling studies with data from He discharges confirmed the mass dependence of the IPB98 $(y, 2)$ scaling, and combined with previous $\mathrm{T}$ and $\mathrm{H}$ studies, suggest $\tau_{E} \propto M^{0.19} Z^{-0.59}$. Threshold power studies in He show the same dependence on magnetic field, density and mass as for deuterium, but the absolute value is about $50 \%$ higher in He. Extensions of the results presented in this paper to higher currents, fields and heating powers and towards longer flat-top durations on JET are prepared, in view

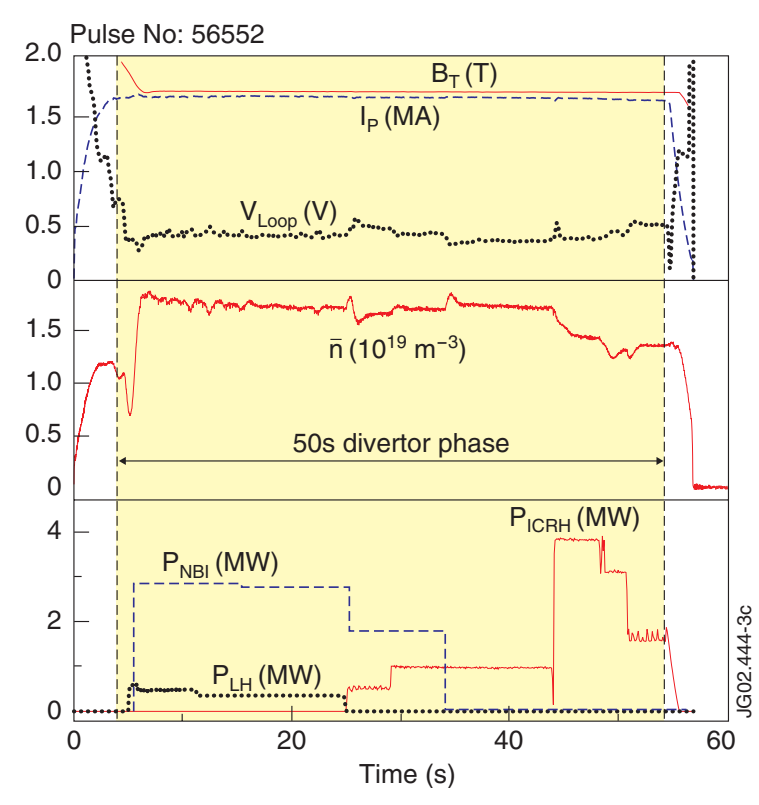

Figure 12. Various plasma parameters of a JET discharge with a $50 \mathrm{~s}$ long divertor phase.

of continued preparation for ITER. New plasma configurations at high average triangularity have been developed, reducing the disruptive forces of the ITER-like configuration, allowing plasma operation at JET up to $4 \mathrm{MA} / 4 \mathrm{~T}$, in order to study the performance at higher absolute densities and to operate closer to $\rho_{*}^{\text {ITER }}$. Such plasmas will require at least $25 \mathrm{MW}$ of additional heating power to reach the H-mode and this level of power should become available during 2003 on JET. Various diagnostics have been and are being enhanced to increase the diagnostic capabilities of JET. A new inboard pellet track aiming more centrally and allowing higher pellet speeds has been installed to further optimize plasma fuelling by high field side pellet injection. Very long pulse scenarios have been prepared with a divertor phase of over $50 \mathrm{~s}$ (figure 12) and with potential for further extensions well over $1 \mathrm{~min}$ [42], presenting a new and interesting tool for studies on the stationarity of various plasma parameters and of recycling during very long time intervals.

\section{References}

[1] Greenwald M. et al 1988 Nucl. Fusion 282199

[2] ITER Physics Basis 1999 Nucl. Fusion 392175

[3] Osborne T.H. et al 2000 Plasma Phys. Control. Fusion 42 A175

[4] Saibene G. et al 1999 Nucl. Fusion 391133

[5] Suttrop W. et al 2000 Plasma Phys. Control. Fusion 42 A97

[6] Kamada Y. et al 1997 Proc. 16th Int. Conf. on Fusion Energy 1996 (Montreal, 1996) (Vienna: IAEA) p 247

[7] Saibene G. et al 2002 Plasma Phys. Control. Fusion 441769

[8] Cordey G. and McDonald D. JET Internal Report http://users.jet.efda.org/pages/codes-data/dvcm/Parameters/ confinement.htm

[9] Lao L.L. et al 2001 Nucl. Fusion 41295

[10] Sartori R. et al 2002 Plasma Phys. Control. Fusion 441801

[11] Ongena J. 2001 Phys. Plasmas 82188

[12] Strachan J.D. et al 2000 Plasma Phys. Control. Fusion 42 A81

[13] Dumortier P. et al 2002 Plasma Phys. Control. Fusion 441845

[14] Puiatti M.E. et al 2002 Plasma Phys. Control. Fusion 441863

[15] Jachmich S. et al 2002 Plasma Phys. Control. Fusion 441879 
[16] Tokar M.Z. et al 2002 Plasma Phys. Control. Fusion 441903

[17] Nave M.F.F. et al 2002 Control of impurity accumulation in JET radiative mantle discharges $\mathrm{Nucl}$. Fusion submitted

[18] Horton L. 1999 Proc. 26th EPS Conf. on Controlled Fusion and Plasma Physics (Maastricht, 1999) paper P1.021 (CD-ROM)

[19] Hillis D. et al 2001 Influence of Ar recycling and divertor configuration on confinement in JET radiating discharges Bull. Am. Phys. Soc. paper GP1.050 (Proc. 43rd Ann. Meeting of the APS Division of Plasma Physics (Long Beach, California, November 2001))

[20] Bateman G. et al 1998 Plasma Phys. 51793

[21] Pereverzev G. et al 2002 Report IPP 5/38, February 2002, IPP-Garching

[22] Erba M. et al 1997 Plasma Phys. Control. Fusion 39261

[23] Bonheure G. et al 2003 Proc. 30th EPS Conf. on Controlled Fusion and Plasma Physics (St. Petersburg, July 2003) Paper P1.100

[24] Jackson G. et al 2002 Plasma Phys. Control. Fusion 441893

[25] Lang P.T. et al 2002 Plasma Phys. Control. Fusion 441919

[26] Nordman H., Weiland J. and Jarmen A. 1990 Nucl. Fusion 30983

[27] Valovic M. et al 2002 Plasma Phys. Control. Fusion 441911

[28] Becoulet M. et al 2002 Plasma Phys. Control. Fusion 44 A103-12

[29] Loarte A. et al 2002 Plasma Phys. Control. Fusion 441815

[30] Fishpool G.M. 1998 Nucl. Fusion 381373

[31] Greenwald M. 1999 Phys. Plasmas 61943
[32] Stober J. et al 2001 Nucl. Fusion 411123

[33] Rapp J. et al 2002 Reduction of heat load in JET ELMy H-modes using impurity seeding techniques Proc. 19th Int. Conf. on Fusion Energy 2002 (Lyon) (Vienna: IAEA) CD-ROM file EX/P1-09, http://www.iaea.org/ programmes/ripc/physics/ fec2002/html/fec2002.htm

[34] Loarte A. et al 2002 Type I ELM energy and particle losses in JET ELMy H-modes and implications for ITER Proc. 19th Int. Conf. on Fusion Energy 2002 (Lyon) (Vienna: IAEA) CD-ROM file EX/P1-08, http://www.iaea.org/ programmes/ripc/physics/fec2002/html/fec2002.htm

[35] Federici G. et al 2003 J. Nucl. Mater. 313-316 11-22

[36] Eich T. 2003 J. Nucl. Mater. 313-316 919-24

[37] Matthews G. 2002 Steady state and transient power handling in JET Proc. 19th Int. Conf. on Fusion Energy 2002 (Lyon) (Vienna: IAEA), CD-ROM file EX/D1-1, http://www.iaea.org/programmes/ripc/physics/fec2002/ $\mathrm{html} / \mathrm{fec} 2002 . \mathrm{htm}$

[38] Cordey G. et al 2002 Plasma Phys. Control. Fusion 441929

[39] Kardaun O.J.F. 1999 Plasma Phys. Control. Fusion 41429

[40] McDonald D. et al 2001 Energy confinement of ELMy $\mathrm{H}$-modes in He-4 plasmas in JET Bull. Am. Phys. Soc. paper Q01.002 (Proc. 43rd Ann. Meeting of the APS Division of Plasma Physics (Long Beach, California, November 2001))

[41] Sauter O. et al 2002 Phys. Rev. Lett. 88105001

[42] Joffrin E. and Lomas P. 2002 personal communication (JET Facilities, Culham Science Centre, 2002) 